\title{
Calidad de vida y enfermedad renal crónica avanzada. Influencia del aclaramiento renal
}

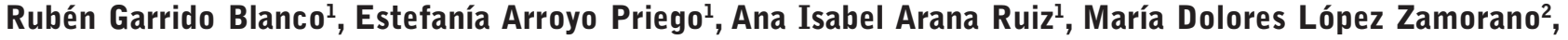 \\ Carmen Tierno Tendero' ${ }^{2}$, Rodolfo Crespo Montero $0^{1,2,3}$ \\ ${ }^{1}$ Departamento de Enfermería. Facultad de Medicina y Enfermería. Universidad de Córdoba. España \\ ${ }^{2}$ Unidad de Gestión Clínica de Nefrología. Hospital Universitario Reina Sofía de Córdoba. España \\ ${ }^{3}$ Instituto Maimónides de Investigación Biomédica de Córdoba. España
}

\section{Resumen}

Objetivos: El objetivo del presente estudio fue analizar la Calidad de Vida Relacionada con la Salud en pacientes con Enfermedad Renal Crónica Avanzada en prediálisis y su relación con el aclaramiento renal.

Material y Método: Se estudiaron 124 pacientes en prediálisis y edad de $67,2 \pm 14,3$ años (45\% mujeres). Se realizó un estudio descriptivo y transversal, en el Servicio de Nefrología de Hospital Reina Sofía de Córdoba. Se utilizó el cuestionario KDQOL-SF, para el análisis de la Calidad de Vida Relacionada con la Salud. También se analizó el aclaramiento renal y la comorbilidad asociada.

Resultados: En el KDQ0L, las dimensiones más afectadas fueron: Situación Laboral, Carga de la Enfermedad Renal y Sueño. Las mujeres presentaron peor puntuación en Listado de Síntomas/problemas. En las dimensiones del SF-36, Salud General, Vitalidad, Función Física y Rol Físico, fueron las más afectadas. Las mujeres obtuvieron peor puntuación en Salud General, Vitalidad, Rol Físico, Dolor y Rol Emocional.

Los pacientes con menor aclaramiento renal presentaron peores puntuaciones en Efectos de la Enfermedad

\section{Correspondencia:}

Rubén Garrido Blanco

C/ Manrique, 21. 4 7.29013 Málaga

E-mail: r.garridoblanco@gmail.com
Renal, Carga de la Enfermedad Renal y Función Sexual; y en Rol Físico y Salud General.

Conclusiones: Los pacientes en prediálisis tienen disminuida su calidad de vida en las dimensiones Carga de la enfermedad renal, Sueño, Salud general, Vitalidad, Función física y Rol físico. El aclaramiento renal, aunque influye directamente en los síntomas derivados de la enfermedad renal, no parece ser tan influyente en la calidad de vida, siendo el sexo femenino, la edad y la comorbilidad asociada, las variables que más se asocian con peor calidad de vida.

PALABRAS CLAVE: ERCA; calidad de vida relacionada con la salud; prediálisis; diálisis.

\section{Quality of life and advanced chronic kidney disease. Influence of renal clearance}

\section{Abstract}

Objectives: The aim of this study was to analyse the Health-Related Quality of Life in patients with Advanced Chronic Kidney Disease in pre-dialysis and the relationship with renal clearance.

Material and Method: We studied 124 patients with ACKD in pre-dialysis and age of $67.2 \pm 14.3$ years (56 women, $45 \%$ ). A descriptive and cross-sectional study was carried out in the Nephrology Service of the Reina Sofía Hospital in Cordoba.The KDQOL-SF questionnaire 
was used to analyse the Health-Related Quality of Life. The renal clearance and associated comorbidity were also analysed.

Results: In the KDQOL, the most affected dimensions were: work situation, burden of kidney disease and sleep. Women presented worse score in list of symptoms / problems. In the dimensions of SF-36, General health, Vitality, Physical function and Physical role were the most affected. Women rated worse scores, significantly, in: General health, Vitality, Physical role, Pain and Emotional role.

Patients with lower renal clearance presented worse scores in Effects of kidney disease, burden of kidney disease and sexual function; and in Physical Role and General Health.

Conclusion: Patients in pre-dialysis have reduced their Health-Related Quality of Life in dimensions Burden of kidney disease, Sleep, General health, Vitality, Physical function and Physical role. Renal clearance, although directly influence the symptoms derived from kidney disease, does not seem to be so decisive in quality of life, being the female sex, age and associated comorbidity, the variables most associated with poorer quality of life.

KEYWORDS: ACKD, health-related quality of life, predialysis, dialysis.

\section{Introducción}

En España, el Estudio Epidemiológico de la Insuficiencia Renal Crónica en España (EPIRCE), señala que la prevalencia global de Enfermedad Renal Crónica (ERC) en estadios 3-5 es del 6,8\%, aumentando esta prevalencia con la edad, independientemente del estadio en el que se encuentran los pacientes ${ }^{1}$. En la última etapa de la ERC (estadio 5), el filtrado glomerular (FG) es inferior a $15 \mathrm{ml} / \mathrm{min} / 1,73 \mathrm{~m}^{2}$, como consecuencia de una lesión en la estructura o función renal, adquiriendo el término de Enfermedad Renal Crónica Avanzada (ERCA); y que inevitablemente, terminará con la inclusión del paciente en alguna Terapia Renal Sustitutiva (TRS) ${ }^{2}$.

Según el Registro de Diálisis y Trasplantes de 2016, de la Sociedad Española de Nefrología, la prevalencia de pacientes con ERCA es de 141,9 pacientes por millón de población ${ }^{3}$. Siendo el grupo de edad más prevalente, el de los enfermos con una edad superior a los 75 años$^{4}$. Se trata, por lo tanto, de una patología que incrementa su prevalencia con la edad, evolucionando inevitablemente hacia la necesidad de recibir una TRS. Esta situación no sólo limita a nivel físico al paciente, sino que además se traduce en una merma de los aspectos más fundamentales de la vida del enfermo, sobre todo a nivel familiar, social, laboral, entre otros ${ }^{5,6}$.

En estrecha relación con esta situación, las personas que padecen ERCA perciben una Calidad de Vida Relacionada con la Salud (CVRS) deteriorada7, así como, una alteración en la realización de las Actividades básicas de la Vida Diaria e Instrumentales (ABVD-AIVD) ${ }^{8}$.

Por otro lado, se ha señalado que el detrimento de la CVRS no sólo viene dado por el deterioro de la función renal producido por la ERCA, sino que además van a influir determinantemente las patologías asociadas, así como las complicaciones derivadas de la enfermedad renal ${ }^{5}$. Teniendo en cuenta todas estas circunstancias, cobran gran importancia los equipos multidisciplinares que desempeñan su labor en las consultas de ERCA, ya que existe evidencia de que un adecuado control de la enfermedad retrasa los problemas asociados a la misma, siendo vital atender y priorizar la mejora de aspectos como la calidad de vida, y no sólo centrarse en los componentes de la enfermedad puramente biológicos ${ }^{9,10}$.

En esta misma línea, se ha destacado que el control precoz en las consultas de ERCA, unido a una correcta praxis de los profesionales sanitarios responsables de la misma, se traduce en una mejora de la CVRS, así como de la consecución de un afrontamiento más adaptativo hacia la enfermedad3,10,11.

Sin embargo, no existe mucha evidencia científica que analice la CVRS en estos pacientes es estadio 5, previa al TRS, es decir, cuando son atendidos en las consultas de Bajo Aclaramiento Renal ${ }^{10}$. Todo lo contrario que ocurre cuando los pacientes ya han sido incluidos en programas de TRS, en los que su calidad de vida ha sido muy estudiada, sobre todo, en los pacientes en diálisisi11,12.

Por esta razón, debida a la importancia que adquiere la percepción de la CVRS en los pacientes con ERCA, sumado a la falta de bibliografía que analice esta variable en los enfermos en fase de prediálisis, es por lo que hemos considerado pertinente el desarrollo de éste trabajo, en un intento de profundizar en las variables que interviene en la calidad de vida de estos pacientes. 
Por todo ello, el objetivo principal del presente estudio fue analizar la CVRS en los pacientes con ERCA en estadios 4-5, y su relación con el aclaramiento renal, con los siguientes objetivos específicos:

1. Determinar la influencia del aclaramiento renal en la CVRS de los pacientes con ERCA.

2. Identificar las variables más influyentes en la calidad de vida de estos pacientes.

\section{Material y Método}

\section{Ámbito del estudio}

El estudio ha sido llevado a cabo en la Unidad de Gestión Clínica de Nefrología del Hospital Universitario Reina Sofía de Córdoba (HURS), en la consulta de Bajo Aclaramiento Renal durante los meses de febrero a mayo de 2018.

\section{Diseño}

Se realizó un estudio observacional descriptivo de corte transversal.

\section{Población y muestra}

La población objeto de estudio han sido las personas con ERCA en estadios 4-5 seguidos en la consulta de Bajo Aclaramiento Renal de la UGC de Nefrología del HURS. El muestreo utilizado fue no probabilístico accidental, dependiendo de las citas programadas en dicha consulta. Una vez fueron identificados los pacientes, se puso en marcha la realización de las entrevistas, a los pacientes que asistieron a consulta, en el periodo de estudio, y que tras haber leído el consentimiento informado y documento de información del estudio, aceptaron participar de forma voluntaria.

Se obtuvo una muestra final de 124 pacientes, con una edad media de $67,2 \pm 14,3$ años; 68 hombres (55\%) y 56 mujeres (45\%). En estadio 4 resultaron 70 pacientes y en estadio 5,54 pacientes.

\section{Variables del estudio}

Variable de resultado: CVRS. Otras variables: FG o aclaramiento renal y comorbilidad, sexo y edad.

\section{Instrumentos de medida}

Como instrumento de medida de la CVRS se empleó el cuestionario validado KDQOL-SF, siendo éste una adaptación del cuestionario SF-36 para pacientes con ERCA.
El citado cuestionario contiene 78 ítems en relación a 19 dimensiones, vinculadas 11 de ellas a escalas específicas para enfermedades renales, como son; síntomas/ problemas, efectos de la enfermedad renal, carga de la enfermedad renal, situación laboral, función cognitiva, calidad de las relaciones sociales, función sexual, sueño, apoyo social, actitud del personal de diálisis y satisfacción del paciente, y 8 ítems del SF-36, correspondientes a: la función física, rol físico, dolor, salud general, bienestar emocional, rol emocional, función social y vitalidad. No fueron cumplimentadas las cuestiones relativas a la satisfacción del paciente y actitud del personal de diálisis, ya que la muestra reclutada no recibían diálisis. Para todas las dimensiones, 100 es la mejor puntación de CVRS ${ }^{13}$.

Para el análisis de la comorbilidad se utilizó el Índice de Comorbilidad de Charlson (ICC). El aclaramiento renal o FG se obtuvo de la base de datos del Servicio de Nefrología. Se dividió a los pacientes en estadio $4\left(F G>15 \mathrm{ml} / \mathrm{min} / 1,73 \mathrm{~m}^{2}\right)$ y estadio $5(F G<15 \mathrm{ml} /$ $\min / 1,73 \mathrm{~m}^{2}$ ).

\section{Recogida de datos}

Los datos fueron recogidos durante el desarrollo de la consulta de Bajo Aclaramiento Renal de la UGC de Nefrología, según la agenda de citación de la misma. Las entrevistas fueron todas realizadas por un único entrevistador, durante el proceso de acto único de la citada consulta, por lo que no hubo necesidad de añadir tiempo adicional de los pacientes, al que rutinariamente permanecen en la consulta.

\section{Aspectos éticos}

La información recogida ha sido tratada con arreglo a la actual Ley Orgánica 15/1999 de 13 diciembre, sobre "Protección de datos de carácter personal", y con el único fin del desarrollo de esta investigación.

El estudio contó con el informe favorable del Comité de Ética e Investigación del HURS (Código TFG-ECVPD-2018).

\section{Análisis estadístico}

Para el análisis de las variables cualitativas se utilizó una distribución de frecuencias, mientras que para las variables numéricas se usó la media \pm la desviación estándar de la media, y en algunos casos la mediana. Para la comparación entre variables se utilizó la $\mathrm{t}$ de Student para muestras independientes. También se realizaron correlaciones con el test de Pearson. Se aceptó significación estadística para $p<0,05$. El análi- 
sis estadístico se ha realizado en un paquete estadístico SSPS 15.0 para Windows.

\section{Resultados}

La media del ICC para la muestra fue de $6,49 \pm 2,8$ puntos. EI ICC fue ligeramente superior en los hombres respecto a las mujeres, aunque sin diferencias estadísticamente significativas.

Los resultados del cuestionario KDQ0L-SF así como los obtenidos para las dimensiones del ceustionario SF-36 se exponen en la Tabla 1.

Tabla 1. Valores de la Escala Específica para Enfermedades Renales y las dimensiones del cuestionario SF-36.

\begin{tabular}{|c|c|c|}
\hline $\begin{array}{l}\text { Escalas Específicas para } \\
\text { Enfermedades Renales }\end{array}$ & Media y DT & Mín-Máx \\
\hline $\begin{array}{l}\text { Listado de } \\
\text { síntomas/problemas }\end{array}$ & $77,5 \pm 16,2$ & $20,45-100$ \\
\hline $\begin{array}{l}\text { Efectos de la enfermedad } \\
\text { renal }\end{array}$ & $80,9 \pm 16,6$ & $25-100$ \\
\hline $\begin{array}{l}\text { Carga de la enfermedad } \\
\text { renal }\end{array}$ & $63,6 \pm 25,2$ & $0-100$ \\
\hline Situación laboral & $31,4 \pm 35,2$ & $0-100$ \\
\hline Función cognitiva & $86,2 \pm 16,3$ & $13,33-100$ \\
\hline $\begin{array}{l}\text { Calidad de las } \\
\text { relaciones sociales }\end{array}$ & $90,2 \pm 14,9$ & $40-100$ \\
\hline Función sexual & $84,2 \pm 28,8$ & $0-100$ \\
\hline Sueño & $74,7 \pm 21$ & $15-100$ \\
\hline Apoyo social & $78,7 \pm 20,8$ & $0-100$ \\
\hline Ítems del SF-36 & Media y DT & Mín-Máx \\
\hline Función física & $52,7 \pm 31,4$ & $0-100$ \\
\hline Rol físico & $55 \pm 46,3$ & $0-100$ \\
\hline Dolor & $67,2 \pm 30,9$ & $0-100$ \\
\hline Salud general & $44,1 \pm 15,9$ & $0-85$ \\
\hline Bienestar emocional & $70,2 \pm 21,3$ & $20-100$ \\
\hline Rol emocional & $72,5 \pm 41,2$ & $0-100$ \\
\hline Función social & $84,9 \pm 22,4$ & $12,50-100$ \\
\hline Vitalidad & $52,3 \pm 25,5$ & $0-100$ \\
\hline
\end{tabular}

Al realizar la comparación hombres y mujeres para la Escala Específica para Enfermedades Renales, encontramos diferencias estadísticamente significativas para la dimensión Listado de síntomas/problemas, y para las dimensiones del cuestionario SF-36, Rol físico, Dolor, Salud general, Rol emocional y Vitalidad, tal como muestra como puede observarse en la Tabla 2.

Tabla 2. Comparación entre hombres y mujeres para las dimensiones del cuestionario KDQOL-SF y el en el SF-36.

\begin{tabular}{|c|c|c|c|}
\hline $\begin{array}{l}\text { Escalas Específicas } \\
\text { para Enfermedades } \\
\text { Renales }\end{array}$ & Hombres & Mujeres & Valor de $\mathbf{p}$ \\
\hline $\begin{array}{l}\text { Listado de } \\
\text { síntomas/problemas }\end{array}$ & $80,5 \pm 15,4$ & $73,8 \pm 16,4$ & $p<0,05$ \\
\hline $\begin{array}{l}\text { Efectos de la } \\
\text { enfermedad renal }\end{array}$ & $82,7 \pm 16,1$ & $78,7 \pm 17,1$ & NS \\
\hline $\begin{array}{l}\text { Carga de la } \\
\text { enfermedad renal }\end{array}$ & $66,3 \pm 22,6$ & $60,3 \pm 27,9$ & NS \\
\hline Situación laboral & $36,7 \pm 37,2$ & $25 \pm 31,6$ & NS \\
\hline Función cognitiva & $87,1 \pm 15,7$ & $85,2 \pm 17,1$ & NS \\
\hline $\begin{array}{l}\text { Calidad de las } \\
\text { relaciones sociales }\end{array}$ & $90,3 \pm 14,4$ & $90,2 \pm 15,7$ & NS \\
\hline Función sexual & $84,7 \pm 28,6$ & $83,7 \pm 29,3$ & NS \\
\hline Sueño & $77,8 \pm 19,1$ & $70,9 \pm 22,8$ & NS \\
\hline Apoyo social & $79,1 \pm 21,6$ & $78,2 \pm 20,1$ & NS \\
\hline Ítems del SF-36 & Hombres & Mujeres & Valor de $p$ \\
\hline Función física & $56,8 \pm 31,9$ & $47,7 \pm 30,2$ & NS \\
\hline Rol físico & $62,5 \pm 45,8$ & $45,9 \pm 45,6$ & $p<0,05$ \\
\hline Dolor & $72,6 \pm 29,3$ & $60,6 \pm 31,8$ & $p<0,05$ \\
\hline Salud general & $47,4 \pm 15,9$ & $40,1 \pm 15,1$ & $p<0,01$ \\
\hline Bienestar emocional & $73,2 \pm 20,9$ & $66,5 \pm 21,5$ & NS \\
\hline Rol emocional & $80,8 \pm 35,1$ & $62,5 \pm 45,8$ & $p<0,05$ \\
\hline Función social & $86,5 \pm 22,5$ & $83 \pm 22,4$ & NS \\
\hline Vitalidad & $57,8 \pm 25,9$ & $45,8 \pm 23,6$ & $p<0,01$ \\
\hline
\end{tabular}

Para comparar los resultados del cuestionario SF-36, respecto a la población general, se utilizaron los valores poblacionales para personas de la misma edad, para este cuestionario (Figura 1).

Para la variable Edad, se hallaron relaciones estadísticamente significativas con las siguientes dimensiones del cuestionario KDQOL-SF: Listado de síntomas/ 


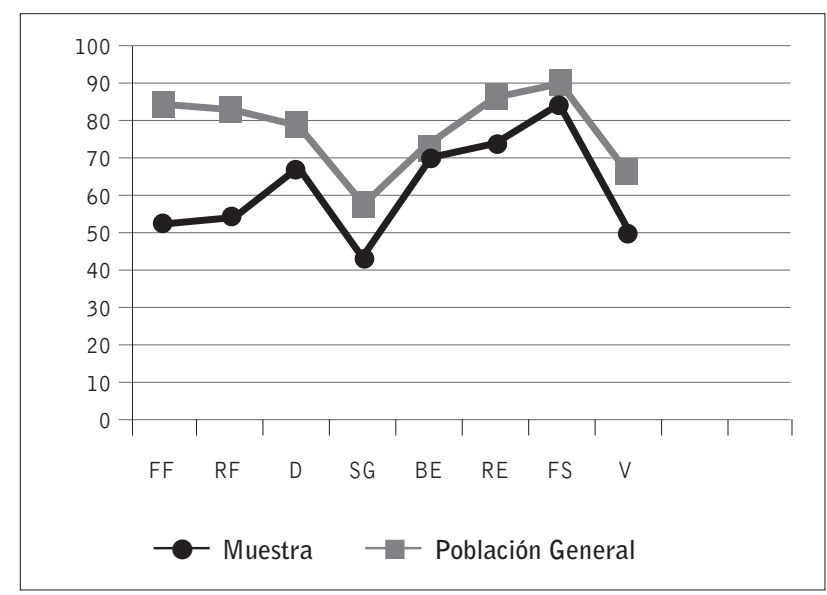

Figura 1. Valores medios de las dimensiones de la población estudiada y la población general española.

problemas $(r=-0,278, p<0,01)$, Situación laboral $(r=-$ $0,199, p<0,05)$ y Función cognitiva $(r=-0,186, p<0,05)$. Mientras que para el cuestionario SF-36, las relaciones significativas para la Edad observadas fueron para las siguientes dimensiones: Función física $(r=-0,480$, $p<0,01)$, Dolor $(r=-0,243, p<0,01)$ y Salud general $(r=-$ $0,222, p<0,05$ ).

Por otro lado, el ICC se relacionó estadísticamente con las dimensiones del cuestionario KDQOL-SF, Listado de síntomas/problemas $(r=-0,209, p<0,05)$, Carga de la enfermedad renal $(r=-0,183, p<0,05)$, Situación laboral $(r=-0,319, p<0,01)$ y Función cognitiva $(r=-0,182$, $<p=0,05)$. Las relaciones significativas halladas para las dimensiones del cuestionario SF-36 fueron, Función física $(r=-0,451, p<0,01)$, Rol físico $(r=-0,183$, $p<0,05)$, Dolor $(r=-0,202, p<0,05)$ y Vitalidad $(r=-$ $0,244, p<0,01)$. Entre las variables Edad e ICC también se encontró relación estadística significativa $(r=0,572$, $p>0,01$ ).

En cuanto al Aclaramiento o FG existe una relación significativa con la dimensión Carga de la enfermedad renal $(r=0,232, p<0,01)$ del cuestionario KDQOL-SF, al igual que para la dimensión Rol físico $(r=0,201$, $p<0,05)$. También observamos una relación significativa entre el Aclaramiento e ICC $(r=0,229, p<0,05)$.

Al comparar los pacientes en estadio 4 frente a los pacientes en estadio 5 , se observó que el grupo con menos aclaramiento presentaba un ICC menor $(5,9 \pm 2,6)$ con respecto al grupo de mayor aclaramiento $(6,9 \pm 2,9)$, aunque la diferencia no fue estadísticamente significativa.
Para el grupo de pacientes de menor aclaramiento renal (estadio 5), la media de edad fue de $67,7 \pm 15,2$, y para el grupo con mayor aclaramiento (estadio 4) la media de edad fue de $66,7 \pm 13,8$ años, sin diferencias significativas.

Cuando se compararon las puntaciones medias entre el grupo de enfermos en estadio 4 y el estadio 5, se encontraron diferencias significativas para las dimensiones Efectos de la enfermedad renal, Carga de la enfermedad renal y Función sexual, para las dimensiones de la escala KDQOL-SF, y en Rol físico y Salud general, para el SF-36, como se representa en la Tabla 3.

Tabla 3. Comparación entre pacientes en estadio 4 y pacientes en estadio 5, para las dimensiones de los cuestionarios KDQOL-SF y SF-36.

\begin{tabular}{|c|c|c|c|}
\hline $\begin{array}{l}\text { Escalas Específicas } \\
\text { para Enfermedades } \\
\text { Renales }\end{array}$ & Estadio 4 & Estadio 5 & Valor de $\mathbf{p}$ \\
\hline $\begin{array}{l}\text { Listado de } \\
\text { síntomas/problemas }\end{array}$ & $79,7 \pm 14,3$ & $74,7 \pm 17,8$ & NS \\
\hline $\begin{array}{l}\text { Efectos de la } \\
\text { enfermedad renal }\end{array}$ & $84,2 \pm 13,5$ & $77,1 \pm 19,3$ & $P<0,05$ \\
\hline $\begin{array}{l}\text { Carga de la } \\
\text { enfermedad renal }\end{array}$ & $71,2 \pm 21,9$ & $54 \pm 25,9$ & $p<0,0001$ \\
\hline Situación laboral & $32,14 \pm 35,1$ & $29,8 \pm 34,6$ & NS \\
\hline Función cognitiva & $88,1 \pm 14,8$ & $84,6 \pm 17,9$ & NS \\
\hline $\begin{array}{l}\text { Calidad de las } \\
\text { relaciones sociales }\end{array}$ & $90,7 \pm 13,2$ & $91 \pm 15,7$ & NS \\
\hline Función sexual & $90,3 \pm 21,5$ & $76,4 \pm 35,2$ & $p<0,001$ \\
\hline Sueño & $76 \pm 21,5$ & $73,6 \pm 20,3$ & NS \\
\hline Apoyo social & $77,6 \pm 20,4$ & $80,1 \pm 21,6$ & NS \\
\hline Ítems del SF-36 & Estadio 4 & Estadio 5 & Valor de $\mathbf{p}$ \\
\hline Función física & $54,7 \pm 31,5$ & $49,4 \pm 31,2$ & NS \\
\hline Rol físico & $68,2 \pm 42,3$ & $37,5 \pm 45,7$ & $p<0,0001$ \\
\hline Dolor & $69,4 \pm 31,5$ & $64,4 \pm 29,8$ & NS \\
\hline Salud general & $46,7 \pm 15,1$ & $40,2 \pm 16,7$ & $p<0,05$ \\
\hline Bienestar emocional & $71,7 \pm 22,1$ & $68,3 \pm 20,1$ & NS \\
\hline Rol emocional & $75,7 \pm 38,8$ & $69,2 \pm 43,7$ & NS \\
\hline Función social & $97,3 \pm 20,1$ & $81,7 \pm 25,4$ & NS \\
\hline Vitalidad & $55,2 \pm 26,2$ & $48,4 \pm 23,9$ & NS \\
\hline
\end{tabular}


También se realizó una comparación entre los pacientes con un ICC inferior a 6 puntos y aquellos con un ICC superior 6 puntos, encontrándose diferencias estadísticamente significativas para los dimensiones, Listado de síntomas/problemas, Efectos de la enfermedad renal y Situación laboral en lo escala KDQOL-SF; y para las dimensiones, Función física, Dolor, Salud general, Bienestar emocional, Función social y Vitalidad, en el SF-36, tal se observa en la Tabla 4.

Tabla 4. Comparación entre los pacientes con ICC $<6$ y los pacientes con ICC $>6$ para las dimensiones de los cuestionarios KDQOL-SF y SF-36.

\begin{tabular}{|c|c|c|c|}
\hline $\begin{array}{l}\text { Escalas Específicas } \\
\text { para Enfermedades } \\
\text { Renales }\end{array}$ & ICC $<6$ & ICC $>6$ & Valor de $\mathbf{p}$ \\
\hline $\begin{array}{l}\text { Listado de } \\
\text { síntomas/problemas }\end{array}$ & $82,2 \pm 14,4$ & $72,4 \pm 16,5$ & $p<0,001$ \\
\hline $\begin{array}{l}\text { Efectos de la } \\
\text { enfermedad renal }\end{array}$ & $83,7 \pm 14,6$ & $77,8 \pm 18,1$ & $p<0,05$ \\
\hline $\begin{array}{l}\text { Carga de la } \\
\text { enfermedad renal }\end{array}$ & $67,6 \pm 26$ & $59,3 \pm 23,9$ & NS \\
\hline Situación laboral & $39,8 \pm 39$ & $22,5 \pm 28,2$ & $p<0,05$ \\
\hline Función cognitiva & $88,5 \pm 15,6$ & $83,9 \pm 16,8$ & NS \\
\hline $\begin{array}{l}\text { Calidad de las } \\
\text { relaciones sociales }\end{array}$ & $91,1 \pm 14,8$ & $89,3 \pm 15,2$ & NS \\
\hline Función sexual & $85,7 \pm 25,6$ & $82,7 \pm 32$ & NS \\
\hline Sueño & $77,9 \pm 20,4$ & $71,3 \pm 21,3$ & NS \\
\hline Apoyo social & $79,6 \pm 20,6$ & $77,7 \pm 21,1$ & NS \\
\hline Ítems del SF-36 & ICC $<6$ & ICC $>6$ & Valor de $\mathbf{p}$ \\
\hline Función física & $65,8 \pm 28,3$ & $38,7 \pm 28,51$ & $p<0,001$ \\
\hline Rol físico & $62,1 \pm 45,2$ & $47,5 \pm 46,7$ & NS \\
\hline Dolor & $75,7 \pm 27,5$ & $58,1 \pm 32$ & $p<0,001$ \\
\hline Salud general & $46,9 \pm 16,4$ & $41 \pm 15$ & $p<0,05$ \\
\hline Bienestar emocional & $74,6 \pm 17,7$ & $65,4 \pm 23,9$ & $p<0,05$ \\
\hline Rol emocional & $76,5 \pm 38,8$ & $68,3 \pm 43,5$ & NS \\
\hline Función social & $89,8 \pm 19$ & $79,8 \pm 24,8$ & $p<0,05$ \\
\hline Vitalidad & $59,6 \pm 25,5$ & $44,5 \pm 23,3$ & $p<0,001$ \\
\hline
\end{tabular}

\section{Discusión}

El objetivo del estudio fue analizar la CVRS en pacientes con ERCA en prediálisis, puesto que se dispone de poca evidencia científica que mida la calidad de vida de estos pacientes. Pese a la falta de dicha bibliografía, sí que encontramos estudios que documentan la calidad de vida de los pacientes con ERCA en TRS 10,14,15.

Para la realización de nuestro estudio, separamos a los pacientes en función del estadio en que se encontraban en el momento del estudio, estadio 4 ( $F G>15 \mathrm{ml} /$ $\left.\mathrm{min} / 1,73 \mathrm{~m}^{2}\right)$ y estadio $5\left(\mathrm{FG}<15 \mathrm{ml} / \mathrm{min} / 1,73 \mathrm{~m}^{2}\right)$, con el objetivo de poder establecer las diferencias y relaciones más significativas para la CVRS de ambos grupos, teniendo en cuenta además, que estos pacientes van a precisar una TRS en un tiempo no muy lejano. De hecho, tanto la edad como la distribución del sexo, de la muestra, es extrapolable a la de la población española en diálisis ${ }^{8,16,17}$.

En cuanto a los resultados, en este grupo de pacientes hemos encontrado una comorbilidad elevada $(6,49 \pm 2,83$ puntos $)$, siendo las patologías más asociadas, las enfermedades cardiovasculares, la diabetes, y el aumento de la edad, estando relacionado todo ello con el aumento de la ERCA ${ }^{1,18,19}$.

En el análisis para las Escalas Específicas para Enfermedades Renales (KDQOL), observamos como dimensiones más afectadas: la Carga de la enfermedad renal, la Situación laboral y el Sueño. Por el contrario, aquellas con mejor resultado fueron: la Función cognitiva y la Calidad de las relaciones sociales, situación que se asemeja a lo publicado en otro estudio ${ }^{20}$. En relación con el apoyo recibido por el entorno próximo del paciente, distintos autores defienden que mejora el afrontamiento de la enfermedad y apuntan a una mejor calidad de vida ${ }^{21,22}$. Aunque la Salud laboral es la dimensión peor puntuada en esta escala, no la destacamos, pues una mayoría de los pacientes eran pensionistas y por tanto, esta puntuación está sesgada, por su edad y situación estar jubilados.

Para el cuestionario SF-36, las dimensiones peor conservadas y que más se alejan de los valores poblacionales de referencia, son la Función física, el Rol físico, la Salud general y la Vitalidad; y las mejor conservadas, el Rol emocional y la Función social. Estos resultados son muy similares a los observados también en pacientes en hemodiálisis ${ }^{23,24,25}$. 
Al comparar hombres y mujeres, observamos que las mujeres obtuvieron peor puntación en el listado de síntomas/problemas en comparación con los hombres en las dimensiones del KDQOL-SF. Además, también en las dimensiones del SF-36, encontramos que las mujeres presentaban peores puntuaciones con respecto a los hombres para: Salud general, Vitalidad, Rol físico, Dolor y Rol emocional. Por el contrario, estudios relacionados con la CVRS de pacientes dializados, apuntaban una peor calidad de vida para la mujer en la dimensión cognitiva, lo que se relaciona con más prevalencia de depresión y ansiedad ${ }^{18,26,27}$.

Por otra parte, observamos que a mayor edad peores puntuaciones en las dimensiones, Listado de síntomas/ problemas, Situación laboral y Función cognitiva del cuestionario KDQOL-SF; y peor puntuación en la Función física, Dolor y Salud general, en las dimensiones del SF-36, resultados estos semejantes a lo reflejado en otro estudio ${ }^{28}$.

Por otro lado, observamos también que a mayor comorbilidad peores puntuaciones en el Listado de síntomas/ problemas, la Carga de la enfermedad renal, la Situación laboral y la Función cognitiva del cuestionario KDQOL-SF. De igual manera, a mayores puntuaciones del ICC, peores puntuaciones en Función física, Rol físico, Dolor y Vitalidad, en el SF-36, observaciones descritas también por otros autores ${ }^{29,30}$.

De todo esto cabe inferir, que tanto la comorbilidad como la edad, tienen una influencia importante en la merma de la calidad de vida de estas personas; $y$ de hecho, al menos en nuestro estudio, existe una buena correlación entre la edad y el ICC $(r=0,572 ; p<0,01)$. Sin embargo, no contamos con evidencia que documente de manera específica estos resultados, ya que los estudios disponibles relacionan ambas variables con una situación de fragilidad que incide en la disminución de la CVRS. Así, algunos autores han destacado, que un aumento de la fragilidad resulta en peores puntuaciones para las dimensiones físicas y mentales, pero no para las sociales ${ }^{31,32}$.

En cuanto al papel desempeñado por el aclaramiento renal en la CVRS, nuestra muestra nos ha permitido comparar esta variable, separando a los pacientes en estadio 4 y estadio 5 . En el cuestionario KDQOL-SF, los pacientes con peor aclaramiento (estadio 5), puntuaron peor en todas las dimensiones del mismo, en comparación con los pacientes en estadio 4, aunque tan sólo, en los Efectos de la enfermedad renal, la Carga de la enfermedad renal y la Función sexual, las diferencias fueron estadísticamente significativas. Como era de esperar, los pacientes con peor aclaramiento renal tenían peor puntuación en aquellas dimensiones relacionadas directamente con la función renal ${ }^{33}$. En el cuestionario SF-36, también los pacientes en estadio 5, puntuaron peor en todas las dimensiones, aunque sólo en el Rol Físico y Salud General, las diferencias fueron significativas. Una posible explicación a esto es que los pacientes en estadio 5 tenían más edad, y está relacionada estadísticamente con estas dos dimensiones, aunque curiosamente, al menos en nuestra muestra, los pacientes en estadio 5 no tenían más comorbilidad que el grupo con mejor aclaramiento, por lo que cabe inferir que estas dos variables no tienen relación, como cabía pensar.

Teniendo en cuenta lo anterior, cobra vital importancia la necesidad de realizar una precoz captación de estos pacientes por parte de los servicios de nefrología, ya que el período prediálisis es crucial para prevenir una empeoramiento posterior de las dimensiones anteriormente citadas y retrasar la merma de calidad de vida que esta enfermedad condiciona9,18,34,35.

La principal limitación del presente estudio ha sido la escasa poca bibliografía existente que analice la CVRS en esta etapa de la ERCA, pues para establecer algún tipo de comparación hemos utilizado estudios de pacientes en diálisis.

A la vista de estos resultados podemos concluir que al menos, en la muestra estudiada, los pacientes con ERCA en estadios 4-5, tienen disminuida su CVRS, con afectación en la mayoría de las dimensiones que engloba este concepto, en comparación con la población sana de referencia. Las dimensiones más afectadas son la Carga de la enfermedad renal, Sueño y Listado de síntomas/problemas en el cuestionario específico de la ERCA; y Salud general, Vitalidad, Función física y Rol físico, en el cuestionario genérico.

El aclaramiento renal, aunque influye directamente en los síntomas derivados de la enfermedad renal, como la Carga y los Efectos de la enfermedad renal, y Función sexual, no parece ser tan influyente en el cuestionario genérico de CVRS. Sin embargo, el sexo femenino, la edad y la comorbilidad asociada, son las variables que más se asocian con peor calidad de vida.

Por último, y dada la escasez de estudios sobre la CVRS en esta etapa de la ERCA, sería conveniente y recomendable, que se llevaran a cabo nuevas investigaciones, para así poder establecer más referencias científicas al respecto. 
Recibido: 1 agosto 2018

Revisado: 15 agosto 2018

Modificado: 7 septiembre 2018

Aceptado: 11 octubre 2018

\section{Bibliografía}

1. Otero A, de Francisco A, Gayoso P, García F. Prevalencia de la insuficiencia renal crónica en España: Resultados del estudio EPIRCE. Nefrología. 2010; 30:78-86.

2. Levey AS, Coresh J. Chronic kidney disease. The Lancet. 2012; 379 (9811):165-80.

3. Registro de Diálisis y Trasplante. Sociedad Española de Nefrología. [citado el 5 de junio de 2017]. Disponible en: http://www.senefro.org/modules.php?na$\mathrm{me}=$ webstructure \&idwebstructure=293.

4. Gutiérrez Sánchez D, Leiva-Santos JP, Sánchez-Hernández R, Gómez García R. Prevalencia y evaluación de síntomas en enfermedad renal crónica avanzada. Enferm Nefrol. 2015;18(3):228-36.

5. Alvarez-Ude F, Rebollo P. alteraciones psicológicas y de la calidad de vida relacionada con la salud en el paciente con enfermedad renal crónica estadios 3-5 (no en diálisis). Nefrología. 2008;28(3):57-62.

6. Brown MA, Collett GK, Josland EA, Foote C, Li Q, Brennan FP. CKD in Elderly Patients Managed without Dialysis: Survival, Symptoms, and Quality of Life. Clin J Am Soc Nephrol. 2015;10(2):260-8.

7. Kaltsouda A, Skapinakis P, Damigos D, Ikonomou M, Kalaitzidis R, Mavreas V, et al. Defensive coping and health-related quality of life in chronic kidney disease: a cross-sectional study. BMC Nephrol. 2011; $12: 28$.

8. Rebollo Rubio A, Morales-Asencio JM, Pons-Raventos ME, Mansilla-Francisco JJ. Revisión de estudios sobre calidad de vida relacionada con la salud en la enfermedad renal crónica avanzada en España. Nefrología. 2015;35:92-09.

9. Karkar A. The value of pre-dialysis care. Saudi J Kidney Dis Transplant Off Publ Saudi Cent Organ Transplant Saudi Arab. 2011;22(3):419-27.

10. Ruiz Parrado M, Gómez Pozo M, Crespo Garrido M, Tierno Tendero $C$ and Crespo Montero R. Análisis de la calidad de vida del paciente en la etapa prediálisis. Enferm Nefrol. 2017;20(3):233-40.

11. Jofre R. Factores que afectan a la calidad de vida en pacientes en prediálisis, diálisis y trasplante renal. Nefrología. 1999;19(1):84-0.

12. Álvarez-Ude F, Vicente E, Badía X. La medida de la calidad de vida relacionada con la salud en los pacientes en programa de hemodiálisis y diálisis peritoneal continua ambulatoria de Segovia. Nefrología. 1995; 15:572-80.

13. Zuñiga C, Dapueto J, Müller H, Kirsten L, Alid R, Ortiz L. Evaluación de la calidad de vida en pacientes en hemodiálisis crónica mediante el cuestionario "Kidney Disease Quality of Life (KDQ0L-36)". Rev Méd Chile. 2009;137:200-07.

14. Seguí Gomá A, Amador Peris $P$, Alcario R, Belén A. Calidad de vida en pacientes con insuficiencia renal crónica en tratamiento con diálisis. Enferm Nefrológica. 2010;13(3):155-60.

15. Tannor EK, Archer E, Kapembwa K, van Schalkwyk SC, Davids MR. Quality of life in patients on chronic dialysis in South Africa: a comparative mixed methods study. BMC Nephrol Lond. 2017;18:4.

16. Ahís Tomás $P$, Peris Ambou IC, Mneu Oset M, Perez Baylach CM, Bonilla Culebras B, Panizo González N. Impacto subjetivo de las estrategias no farmacológicas de mejora de la calidad de vida y el cumplimiento terapéutico en pacientes en hemodiálisis. Enferm Nefrológica. 2017;20(1):22-7.

17. Costa G, Pinheiro M, Medeiros S, Costa R, Cossi M. Calidad de vida en pacientes con insuficiencia renal crónica en hemodiálisis, Enferm Global. 2016;15 (43):59-73.

18. Rebollo Rubio A, Morales Asensio JM, Pons Raventos ME. Influencia de la consulta de enfermería de enfermedad renal crónica avanzada en pacientes que inician tratamiento renal sustitutivo. Enferm Nefrol. 2014; 17(4):243-50. 
19. Snively CS, Gutierrez C. Chronic Kidney Disease: Prevention and Treatment of Common Complications. Am Fam Physician. 2004;70(10):1921-8.

20. Poersch RF, Andrade FP, Bosco AD, Edit Rovedder PM. Quality of life of chronic renal failure patients undergoing hemodialysis. ConScientiae Saúde Sao Paulo. 2015;14(4):608-16.

21. Ibrahim N, Teo SS, Din NC, Gafor AH, Ismail R. The Role of Personality and Social Support in Health-Related Quality of Life in Chronic Kidney Disease Patients. PLoS One. 2015;10(7).

22. Kara B, Caglar K, Kilic S. Nonadherence with diet and fluid restrictions and perceived social support in patients receiving hemodialysis. J Nurs Scholarsh Off Publ Sigma Theta Tau Int Honor Soc Nurs. 2007; 39(3):243-8.

23. Adell Lleixà $M$, Casadó Marin $L$, Andújar Solé J, Solà Miravete E, Martínez Segura E, Salvadó Usach T.Valoración de la calidad de vida relacionada con la salud en la enfermedad renal crónica terminal mediante un cuestionario de resultados percibidos por los pacientes. Enferm Nefrológica. 2016; 19(4):331-40.

24. Frías Albañil T, Ramírez Moreno MC, Crespo Montero R. Análisis de la calidad de vida en pacientes eh hemodiálisis ambulatoria y su relación con el nivel de dependencia. Enferm Nefrol. 2014;17(3):167-74.

25. Pagels AA, Söderkvist BK, Medin C, Hylander B, Heiwe $S$. Health-related quality of life in different stages of chronic kidney disease and at initiation of dialysis treatment. Health Qual Life Outcomes. 2012;10:71.

26. García-Llana H, Remor E, Peso G del, Selgas R. El papel de la depresión, la ansiedad, el estrés y la adhesión al tratamiento en la calidad de vida relacionada con la salud en pacientes en diálisis: revisión sistemática de la literatura. Nefrología. 2014;34(5):637-57.

27. Sanz Guajardo D, López Gómez JM, Jofre R, Fort R, Valderrábano $F$, Moreno F, Vázquez I. Diferencias en la calidad de vida relacionada con la salud entre hombres y mujeres en tratamiento en hemodiálisis. Nefrología. 2004;24:167-78.

28. Santos PR. Relationship between gender and age with quality of life in chronic hemodialysis patients. Rev Assoc Médica Bras. 2006; 52(5):356-9.

29. Cruz MC, Andrade C, Urrutia M, Draibe $S$, Nogueira-Martins LA, de Castro Cintra Sesso R. Quality of life in patients with chronic kidney disease. Clinics. 2011; 66(6):991-5.

30. Castro M, Caiuby AVS, Draibe SA, Canziani MEF. Qualidade de vida de pacientes com insuficiência renal crônica em hemodiálise avaliada através do instrumento genérico SF-36. Rev Assoc Med Bras. 2003;49:245-9.

31. Mansur HN, Colugnati FA, Grincenkov FR dos S, Bastos MG. Frailty and quality of life: a cross-sectional study of Brazilian patients with pre-dialysis chronic kidney disease. Health Qual Life Outcomes. 2014; 12:27.

32. Lee SJ, Son $H$, Shin SK. Influence of frailty on health-related quality of life in pre-dialysis patients with chronic kidney disease in Korea: a cross-sectional study. Health Qual Life Outcomes. 2015;13:70.

33. Legrand K, Speyer E, Ayav C, Ngueyon Sime W, Stenguel B, Briançon S. État de santé et qualité de vie perçu des patients selon le stade de la maladie rénale chronique. 2016;12(5).

34. Sakhuja A, Hyland J, Simon JF. Managing advanced chronic kidney disease: a primary care guide. Cleve Clin J Med. 2014;81(5):289-99.

35. Forero Villalobos J, Barrios Araya S. Rol de enfermería en la consulta de prediálisis en el paciente con enfermedad renal cónica avanzada. Enferm Nefrológica. 2016;19(1):77-86.

Este artículo se distribuye bajo una Licencia Creative Commons Atribución-NoComercial 4.0 Internacional. https://creativecommons.org/licenses/by-nc/4.0/

Open Access (c) () 